\title{
Vestibular effects of diving
}

\author{
M. Milkov', M. Stoykov', S. Peev', D. Petrova² \\ 'Faculty of Dental medicine, Medical University "Prof. Dr. Paraskev Stoyanov" - Varna \\ "Medical University "Prof. Dr. Paraskev Stoyanov" - Varna
}

\begin{abstract}
Over the last 45 years, recreational self-contained underwater breathing apparatus (SCUBA) diving as a leisure activity has increased in popularity. According to the Professional Association of Diving Instructors (PADI), more than 23 million diver certificates have been issued across the globe. Given the popularity of scuba diving, it is incumbent on every physician to know and understand the specific medical hazards and conditions associated with scuba diving. Although diving has become more accessible and the equipment is much safer now, diving still has its inherent dangers. Each year incidents occur, ranging from seasickness and sinus problems to heart conditions and mortality. More than $80 \%$ of all diving complications occur in the head and neck region. A number of studies have found that diving can lead to the development of vestibular disorders such as: Barotrauma, Decompression sickness, Otitis externa, Bilateral exostoses, Chronic sinusitis (barosinusitis), Serous tubotympanic catarrh, Tinnitus, Dizziness, Vertigo.

The aim of this paper was to share our experience in the field of treating patients with different types of ENT disorders after or before a diving session. We briefly summarized the most important information for some of the disorders.

We included in our study for a period of 12 years: a total of 24 patients (four females and 20 males), who dive. They were between 25 and 55 years of age. Some presented in clinics with an ENT disorder. 19 were professional, qualified divers, five (three males and two females) dove for the first time. On all 24 patients we performed: audiometry tests, Tympanometry tests, OAE, Static and dynamic vestibular tests, vHIT - in the last five years, otoscopy examination, dental check-ups.

All divers - no matter professionalists or not - should follow these recommendations: a thorough prophylactic ENT check-up should be performed before an active diving session. Chronic ENT inflammations should be addressed in a timely manner. Divers should follow all instructions from the certified Diving Associations.
\end{abstract}

Key words: ENT disorders, SCUBA diving, vestibulopathy, PADI

\section{Introduction}

Over the last 45 years, recreational self-contained underwater breathing apparatus (SCUBA) diving as a leisure activity has increased in popularity. The ability to explore the ocean depths is an obvious attraction for many. According to the Professional Association of Diving Instructors (PADI), more than 23 million diver certificates have been issued across the globe, with a mean 900,000 certificates in more than 200 countries issued annually. (12) And they even grow more. Roughly two-thirds of these certified divers are men (mean age: men, 30 years; women, 27 years). Given the popularity of scuba diving, it is incumbent on every physician to know and understand the specific medical hazards and conditions associated with scuba diving. Although diving has become more accessible and the equipment is much safer now, diving still has its inherent dangers. Each year incidents occur, ranging from seasickness and sinus problems to heart conditions and mortality. More than $80 \%$ of all diving complications occur in the head and neck region. Of these, approximately $65 \%$ are outer, middle or inner ear disorders. (11)

The aim of this paper was to share our experience in the field of treating patients with different types of ENT disorders after or before a diving session and to briefly summarize the most important information for some of the disorders. 


\section{Material and methods}

We included in our study for a period of 12 years: a total of 24 patients (four females and 20 male), who dive. They were between 25 and 55 years of age (mean: 44.5). Some presented in clinics with an ENT disorder. 19 were professional, qualified divers, five (three male and two female) dove for the first time. Two patients (one male and one female) from the group of the non-professional divers developed a strong episode of vertigo after leaving the water. One patient (male) from the group of the professional divers developed acute serous otitis of the middle ear and acute vestibular reactions. Of all 24 patients, divers, five had chronic sinusitis, two had serous tubotympanic catarrh, one had tinnitus, three had a general vestibulopathy - dizziness and rare episodes of vertigo, 15 were healthy. On all 24 patients we performed: audiometry tests (Audiogram), Tympanometry tests (Tympanogram), OAE, Static and dynamic vestibular tests, vHIT - in the last five years, otoscopy examination, dental checkups. Four of the chronic sinusitis patients (a total of five) underwent surgical treatment - septoplasty or Functional Endoscopic Sinus Surgery (FESS). So as to summarize the information for some of the diseases, we searched through MEDLINE, PubMed, Scopus and other scientific databases, using the time period 1975-2020. The following keywords were used: ENT disorders, SCUBA diving, vestibulopathy, PADI.

\section{Discussion}

A number of studies have found that diving can lead to the development of vestibular disorders such as: Barotrauma, Decompression sickness, Otitis externa, Bilateral exostoses, Chronic sinusitis (barosinusitis), Serous tubotympanic catarrh, Tinnitus, Dizziness, Vertigo. (1), (2), (3), (5), (6), (13)

Barometric pressure at sea level is $100 \mathrm{kPa}$ and increases linearly underwater with every 10 meters of descent, adding $100 \mathrm{kPa}$. As depth increases, the diver breathes gas of increased density while the diving equipment provides oxygen and allows for elimination of carbon dioxide. Increased depth and pressure also leads to a decrease in gas volume and an increase in the amount of gas dissolved in body tissues. The otologic conditions of barotrauma and decompression sickness that can result from scuba diving are related to the behavior of gas changing under pressure as the diver descends. Barosinusitis is a disease of sinonasal injury and inflammation 34 due to rapid, uncompensated changes of pressure within the sinonasal anatomy, for example when diving. That leads to mucosal damage, most commonly resulting in facial pain or pressure over the affected sinuses (92\%), headache, odontalgia, cloudy mucus, and more severely, epistaxis. Out of the paranasal sinuses, the paired frontal sinuses are most commonly affected followed by the maxillary sinuses, and more rarely the sphenoid sinuses. There are no reported cases of ethmoid barosinusitis likely due to their location and surrounding anatomical configuration. Risk factors for sinus barotrauma when underwater diving include any etiology that would narrow or obstruct the natural sinus ostia. These etiologies include but are not limited to nasal polyposis; stenosis of sinus ostia; obstruction with mucous or fungus ball or sinusitis. (4), (7), (15), (16), (17), (18), (21), (25).

Otitis externa (OE) is the most common otologic disorder among divers, afflicting nearly half of all active divers on at least one occasion. Pseudomonas aeruginosa is the most common micro-organism responsible. Treatment is no different among divers versus non-divers and should consist of dry ear precautions, topical antimicrobial therapy and serial debridement of the external auditory canal. Divers should be counselled on the prevalence of this condition and may consider bringing antimicrobial drops on remote diving trips. (8), (22)

Exostoses are bony outgrowths of the external auditory canal (EAC) that typically occur bilaterally in scuba divers. They are very common, with a prevalence of up to $40 \%$ among professional divers. Exostoses can lead to recurrent otitis externa, recurrent otorrhoea, cerumen impaction and conductive hearing loss from canal obstruction. If the canal is completely occluded, an asymmetrical caloric stimulus with vertigo can occur upon submersion. Divers with symptomatic exostoses should seek evaluation by an experienced otolaryngologist for consideration of canalplasty. Surgical indications for removal are identical to non-divers, with the caveat that they are very likely to experience progression of their exostoses due to further water exposure, and are at higher risk of OE. Exostoses should not affect fitness to dive unless they are occluding the canal or causing recurrent infections. (9), (23), (24)

Serous tubotympanic catarrh (otitis media with effusion) is a long-known disease. Today it has become a "fashionable disease", but is extremely important as the most frequent cause of hearing loss in 
children. The key to the disease is functional disability of the auditory tube. (8), (19)

Tinnitus is a perception of spontaneous noises within the head or ears with no external source. Apparently generated within the individual's auditory pathway, its precise cause is unknown. Although tinnitus is one of the symptoms that can be found in divers, little research specifically investigates tinnitus associated with diving, except for some examination of navy divers. Among divers, the symptom is normally connected with hearing loss and vertigo. (8), (26)

Vertigo can be described as the hallucination of movement and/or the sensation that patient's surroundings are moving. Diving conditions can interfere with the visual cues available, due to decreased visibility as well as the feeling of weightlessness that will cause a loss of proprioceptive input. Therefore, reliance on vestibular input is greatly increased underwater and any sudden encounter of vertigo will cause an increased safety risk to the diver. (8), (14), (27)

\section{Conclusion}

All divers - no matter professionalists or not should follow these recommendations: a thorough prophylactic ENT check-up should be performed before an active diving session. Chronic ENT inflammations should be addressed in a timely manner. If a vestibular pathology is observed - vHIT, nystamography, VEMP should be performed. Divers should follow all instructions from the certified Diving Associations - Professional Association of Diving Instructors (PADI).

The presented article was a part of the $14^{\text {th }}$ National congress of otorhinolaryngology - quality in the otorhinolaryngology, despite the routine, 2021.11.2020 - virtual presentation on 21.11.2020 09:30.

\section{References:}

1. Livingstone DM, Smith KA, Lange B. Scuba diving and otology: a systematic review with recommendations on diagnosis, treatment and post-operative care. Diving Hyperb Med. 2017;47(2):97-109. doi:10.28920/dhm47.2.97-109

2. Glazer TA, Telian SA. Otologic Hazards Related to Scuba Diving. Sports Health. 2016;8(2):140-144. doi:10.1177/1941738116631524

3. Evens RA, Bardsley B, C Manchaiah VK. Auditory complaints in scuba divers: an overview. Indian J Otolaryngol Head Neck Surg. 2012;64(1):71-78. doi:10.1007/s12070-011-0315-6

4. Klingmann C, Praetorius M, Baumann I, Plinkert PK. Barotrauma and decompression illness of the inner ear: 46 cases during treatment and follow-up. Otol Neurotol. 2007;28:447-54. doi: 10.1097/MAO.0b013e318030d356. PubMed PMID: 17417111

5. Roydhouse N. 1001 disorders of the ear, nose and sinuses in scuba divers. Can J Appl Sport Sci. 1985;10:99-103. PubMed PMID: 4017159.

6. Edmonds CB, Lippmann, Mitchell S, editors. Diving and subaquatic medicine. Boca Raton, FL: CRC Press; 2015.

7. Beckett A, Kordick MF. Risk factors for dive injury: a survey study. Res Sports Med. 2007;15:201-11. doi: 10.1080/15438620701526779. PubMed PMID: 17987508.

8. Azizi MH. Ear disorders in scuba divers. Int J Occup Environ Med. 2011;2:20-6. PubMed PMID: 23022815

9. House JW, Wilkinson EP. External auditory exostoses: evaluation and treatment. Otolaryngol Head Neck Surg. 2008;138:672-8. doi: 10.1016/j.otohns.2008.01.023. PubMed PMID: 18439477.

10. DiBartolomeo J. Exostoses of the external auditory canal. Ann Otol Rhinol Laryngol. 1979;88(Suppl 61):2-20.

11. Diver's Alert Network. Surfer's Ear. [cited 2017 January 07]. Available from: https://www.diversalertnetwork.org/health/ears/surfers-ear.

12. PADI. Wordwide corporate statistics 2015. 2015. http://www.padi.com/scubadiving/about-padi/statistics/. Accessed September 1, 2015.

13. Edmonds C, Lowry C, Pennefather J, Walker R (2005) Diving \& sub-aquatic medicine, 4th edn. Hodder Headline Group, London

14. NHS Direct (2009) Vertigo. Available at: http://www.nhsdirect.wales.nhs.uk/small/en/home/healthencyclopaedia/v/vertigo

15. Battisti AS, Lofgren DH, Lark JD. Barosinusitis. [Updated 2020 Jul 10]. In: StatPearls [Internet]. Treasure Island (FL): StatPearls Publishing; 2020 Jan-. Available from: https://www.ncbi.nlm.nih.gov/books/NBK470207/

16. Vaezeafshar R, Psaltis AJ, Rao VK, Zarabanda D, Patel ZM, Nayak JV. Barosinusitis: Comprehensive review and proposed new classification system. Allergy Rhinol (Providence). 2017 Oct 01;8(3):109-117.

17. Jamil RT, Reilly A, Cooper JS. StatPearls [Internet]. StatPearls Publishing; Treasure Island (FL): Aug 14, 2020. Sinus Squeeze (Barosinusitis, Aerosinusitis)

18. Gunepin M, Derache F, Blatteau JE, Nakdimon I, Zadik Y. Incidence and Features of Barodontalgia Among Military Divers. Aerosp Med Hum Perform. 2016 Feb;87(2):137-40.

19. Erlach A, Zechner G. Der Tubenmittelohrkatarrh im Kindesalter - Diagnose und Therapie [Tubotympanic catarrh in childhood. Diagnosis and therapy]. Wien Klin Wochenschr. 1983 Jun 10;95(12):396-9. German. PMID: 6613135.

20. https://www.haqqitours.com/fethiye-tours/scuba-diving-fethiye/

21. https://www.diversalertnetwork.org/diving-incidents/unrecognized ear barotrauma

22. https://www.aboutkidshealth.ca/swimmersear

23. https://www.tuiearclinic.co.nz/surfers-ear

24. https://www.surfline.com/surf-news/surfers-ear-explained-everything-you-need-to-know-about-exostosis/17888

25. http://impocity.blogspot.com/2015/03/aerosinusitis-or-barosinusitis.html

26. https://www.mayoclinic.org/diseases-conditions/tinnitus/symptoms-causes/syc-20350156

27. https://www.mountnittany.org/articles/healthsheets/4128 